deseriptions of the actual routes between release points and home loft.

From this investigation and those of other workers, Griffin concludes that it is advisable to distinguish three distinct levels of navigational ability. What may conveniently be called type $I$ homing is reliance upon "contact flying" within familiar territory. When released in totally unfamiliar territory, birds which exhibit only type I homing show a quantitative homing performance that can easily be accounted for by theories of exploration or even by a completely random search for familiar landmarks. They may be misled by topographic features resembling portions of their familiar territory.

A second level of homing, conveniently designated type II, is the ability to maintain flight in roughly a fixed direction, usually that adhered to in previous training flights.

There is also a third level of homing ability, conveniently designated as type III, which permits birds to fly approximately straight towards home from unknown territory regardless of the direction in which home lies.

While differentiating between these three types, the same flock of pigeons may exhibit two, or even all three, of these types of orientation at different times during a single homing flight.

Finally, it must be admitted that the central problem of pigeon navigation remains unsolved; the sensory basis of orientation in both type II and type III homing is still a matter for speculation. The results achieved to date point towards a sort of celestial navigation based upon the sun and sky brightness patterns.

\section{VARIATION OF HUMIDITY WITH HEIGHT IN THE LOWER ATMOSPHERE}

$\mathrm{D}$ URING the Second World War, a need arose for detailed information on the variation of humidity with height in the lower layers of the atmosphere in connexion with research into the refraction of the electromagnetic waves used in radar and high-frequency radio communication. The termperature structure in the vertical and its variations were known in considerable detail from work at Porton and Leafield, by Sir Nelson Johnson and Mr. G. S. P. Heywood, published in Geophysical Memoirs 46 and 77 , but no such information existed for humidity. To remedy the deficiency the Meteorological Office set up apparatus near Rye, Sussex, recording temperature and humidity at $1 \cdot 1 \mathrm{~m}$. and on an open-lattice mast at heights of $15 \cdot 2,47 \cdot 2$ and $106.7 \mathrm{~m}$. Temperature was recorded continuously by resistance thermometers and relative humidity by Gregory humidiometers enclosed in aspirated screens.

Geophysical Memoir 89* describes the apparatus in detail and summarizes and discusses the observations made at Rye during July 1945-June 1948. The temperature observations at Rye differed little in their main properties from those made by Johnson and Heywood, and in this note attention will be concentrated on the humidity. Humidity is expressed

* Temperature and Humidity Gradients in the First $100 \mathrm{~m}$. over South-East England, By A. C. Best, E. Knighting, R. H. Pedlow and K. Stormonth. (Air Ministry : Meteorological Office, Geophysical Office, 1952.) $78.6 d$. net. in the Memoir in milligrams of water vapour per cubic metre; mean values of this quantity at $1 \cdot 1 \mathrm{~m}$. are about 11,000 in midsummer and 5,000 in midwinter.

The variation of humidity with height is similar to the variation of temperature with height. There is generally a decrease (lapse) with height in the middle of the day and an increase (inversion) at night. The largest variations occur near the ground. The vertical gradient decreases with height.

The diurnal variations are of great interest, especially on clear days in summer when, as would be expected, they are most pronounced. Humidity near the ground on clear days increases quickly after sunrise as insolation evaporates water from the ground surface, and there is a sharp maximum two to three hours later. A lapse of humidity forms and quickly becomes of the order of $1,000-1,500 \mathrm{mgm} . / \mathrm{m}^{3}$ between $1 \cdot 1 \mathrm{~m}$. and $106 \cdot 7 \mathrm{~m}$. Increasing turbulence then transports water vapour upward more quickly than it is evaporated so that humidity near the ground and the lapse-rate decrease again. In the evening turbulence decreases, so humidity near the ground increases again, until after sunset the lapse of humidity changes over to an inversion and water vapour diffuses down to condense on the ground. There is a minimum of humidity and the most pronounced inversion at dawn; an increase of some $800 \mathrm{mgm} . / \mathrm{m}^{3}$ between $1.1 \mathrm{~m}$. and $106.7 \mathrm{ma}$. is then observed. After sunrise the inversion breaks down very quickly as evaporation begins.

The Memoir concludes with a description of the temperature and humidity gradients observed in radiation fog. The general picture is of an inversion of both temperature and humidity in the lowest layers in the early stages of fog. These inversions weaken in low layers as the air cools further and the depth of fog increases but extend upwards to continually increasing heights. Relative humidity inside the fog remains almost constant but need by no means reach 100 per cent.

The Memoir reports an investigation of fundamental importance in meteorology and will undoubtedly be consulted as much as its similar predecessors, Nos. 46 and 77.

\section{HISTOCHEMISTRY OF XANTHINE OXIDASE}

By Dr. GEOFFREY H. BOURNE

Histology Department, London Hospital Medical College

$T^{T}$ was shown by Shelton and Schneider ${ }^{1}$ that a 1 mixture composed of purified xanthine oxidase, hypoxanthine and phosphate buffer at a $p H$ of $7 \cdot 5$, when incubated with neotetrazolium, reduced the latter to an insoluble coloured diformazan. The reaction was similar to that produced by succinic dehydrogenase in the presence of succinate. Since the latter enzyme has been demonstrated histochemically by this method (Seligman and Rutenberg ${ }^{2}$ ), it was decided to attempt the histochemical localization of xanthine oxidase in a similar way.

A substrate mixture containing $3 \times 10^{-4} M$ xanthine in $0.075 M$ sodium pyrophosphate buffer, giving a $p H$ of about $8 \cdot 2$, was used and to this was added $50 \mathrm{mgm}$. of neotetrazolium chloride. Nitrogen was bubbled through this mixture for $20 \mathrm{~min}$., and it 